TITLE:

\title{
Investigation of magnetic characteristics in HTS bulk materials for motor applications
}

$\operatorname{AUTHOR}(S)$ :

Nakamura, T; Fukui, K; Jung, HJ; Muta, I; Hoshino, T

\section{CITATION:}

Nakamura, T ... [et al]. Investigation of magnetic characteristics in HTS bulk materials for motor applications. IEEE TRANSACTIONS ON APPLIED SUPERCONDUCTIVITY 2003, 13(2): 2255-2258

ISSUE DATE:

2003-06

URL:

http://hdl.handle.net/2433/39952

\section{RIGHT:}

(c)2003 IEEE. Personal use of this material is permitted. However, permission to reprint/republish this material for advertising or promotional purposes or for creating new collective works for resale or redistribution to servers or lists, or to reuse any copyrighted component of this work in other works must be obtained from the IEEE. 


\title{
Investigation of Magnetic Characteristics in HTS Bulk Materials for Motor Applications
}

\author{
Taketsune Nakamura, Kentaro Fukui, Hun-June Jung, Itsuya Muta, and Tsutomu Hoshino
}

\begin{abstract}
Magnetic characteristics of $\mathrm{Bi}-2223$ bulk rotor that was used for the HTS motor was investigated at $77.3 \mathrm{~K}$. Spatial distribution of the magnetic flux density in the air-gap of axial-type HTS motor was measured in a rotating magnetic field at $60 \mathrm{~Hz}$ frequency. Numerical simulation based on 3D finite element method was also performed. It was shown that the pinning property of the bulk rotor influences the harmonic components of the air-gap flux density as well as motor characteristics.
\end{abstract}

Index Terms-Bi-2223, bulk motor, finite element method, spatial harmonics.

\section{INTRODUCTION}

INCE the discovery of high- $T_{c}$ supesconductor (HTS) and $\checkmark$ the following development of high quality HTS bulk, extensive and intensive efforts have been made for the realization of power application systems using bulk materials. As one of such applications, some kinds of HTS bulk motors, i.e., hysteresis type, reluctance type, and trapped-field type, have been proposed and studied in order to increase power density as well as efficiency compared to conventional normal conducting motors [1]-[5]. Further, some specific applications such as cryogenic pump for the circulation of liquid fuel have also been considered [6].

YBCO bulk materials have usually been used as the rotor because of the strong pinning characteristics at 77.3 K [7]-[10]. On the other hand, we have been considering the Bi-2223 bulk rotor for the applicability study as well as academic interests. However, it has already been shown experimentally that the fabricated $\mathrm{Bi}-2223$ bulk motor is operated with large slip mode and shows the complicated characteristics including hysteretic and induction torques [11]-[14]. There are mainly two reasons for these motor characteristics, i.e., spatial harmonic components of the magnetic flux distribution in the air-gap and pinning property of the Bi-2223 bulk material. That is, asynchronous torque is induced by the spatial harmonic components, then slippage occurs [14], [15]. With respect to these problems, detailed experimental study will also be presented in [16].

In this paper, the effect of pinning characteristics of $\mathrm{Bi}-2223$ rotor upon the spatial harmonics of the magnetic flux distribution is investigated. Magnetic flux density in the air-gap of the

Manuscript received August 6, 2002. This work was supported in part by Grant-In-Aid for Scientific Research 13450113 from the Ministry of Education, Culture, Sports, Science and Technology, Japan.

The authors are with the Graduate School of Engineering, Kyoto University, Kyoto 606-8501, Japan (e-mail: tk_naka@kuee.kyotou.ac.jp; k-fukui@asl.kuee.kyoto-u.ac.jp; hjjung@asl.kuee.kyoto-u.ac.jp; muta@kuee.kyoto-u.ac.jp; hoshino@asl.kuee.kyoto-u.ac.jp).

Digital Object Identifier 10.1109/TASC.2003.813059

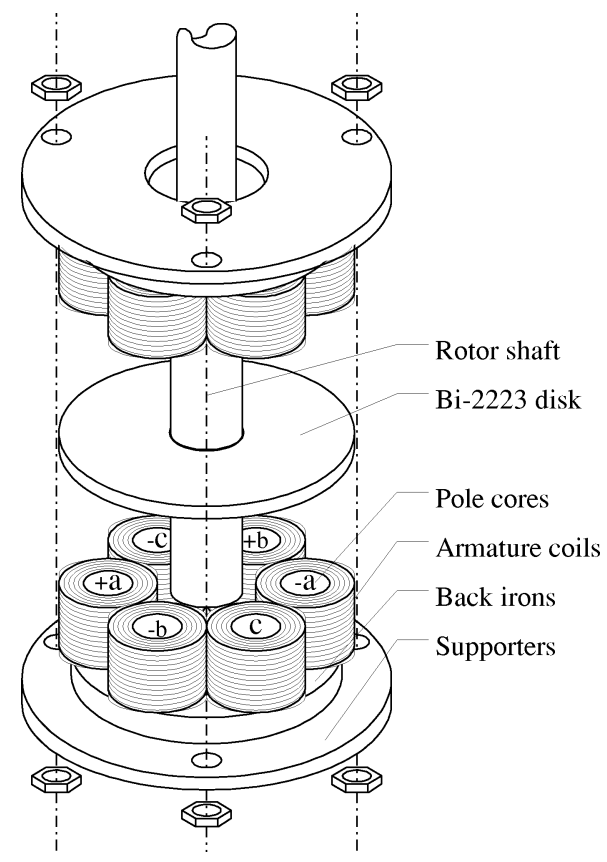

Fig. 1. Schematic diagram of axial-type Bi-2223 bulk motor. 3-phase and 2-pole armature windings are used for the generation of rotating magnetic field.

fabricated motor is measured in a rotating magnetic field (60 $\mathrm{Hz}$ frequency) at room temperature and $77.3 \mathrm{~K}$. Simulation of electromagnetic properties based on 3-D finite element method is also performed.

\section{EXPERIMENTAL METHOD}

Schematic diagram of the fabricated $\mathrm{Bi}-2223$ bulk motor is shown in Fig. 1. 3-phase and 2-pole salient armature windings are used for the application of rotating magnetic field at $60 \mathrm{~Hz}$ frequency. $\mathrm{Bi}_{1.85} \mathrm{~Pb}_{0.35} \mathrm{Sr}_{1.90} \mathrm{Ca}_{2.05} \mathrm{Cu}_{3.05} \mathrm{O}_{x}$ ( $\left.\mathrm{Bi}-2223\right)$ bulk, $120 \mathrm{~mm}$ in outer diameter, $26 \mathrm{~mm}$ in inner diameter and $7 \mathrm{~mm}$ in thickness, is used for the rotor of the motor. Detailed structure and the explanations of the fabricated motor are shown in [13]-[15]. Fig. 2 shows the experimental circuits for the mesurement of air-gap magnetic flux density in a rotating magnetic field. The air-gap magnetic flux density is measured by transverse type hall sensor which is mounted on the surface of the bulk rotor and the concentric line which lie on the center of each armature coil. Hall signal as well as the phase current of phase-a is recorded by the transient recorder as a function of time. Air-gap length between the surface of armature coil and that of the bulk rotor is set to be $1.5 \mathrm{~mm}$. The measurements are performed at angular intervals of $1^{\circ}$. 


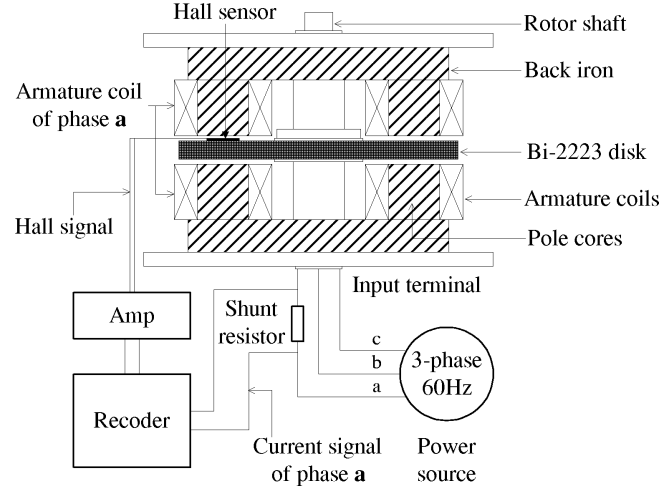

Fig. 2. Experimental circuit for the measurement of air-gap magnetic flux density. Magnetic flux density is measured in a rotating magnetic field $(60 \mathrm{~Hz})$ that is applied by 3-pahse, 2-pole armature windings.

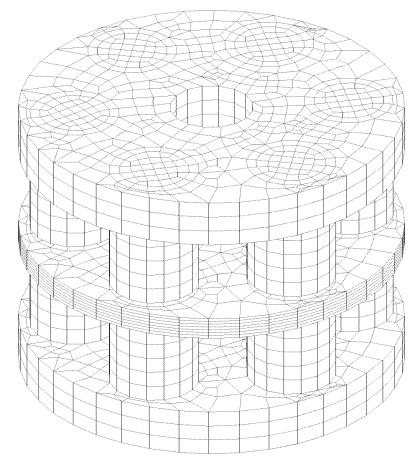

Fig. 3. FEM model constructed for Fig. 1.

\section{Simulation MethoD}

Simulation of electromagnetic property in the motor system is performed based on 3 dimensional finite element method (3D-FEM). Fig. 3 shows the analysis model constructed for Fig. 1. The constitutive relation of electric field, $E$, vs. current density, $J_{\mathrm{sc}}$, in superconducting Bi-2223 bulk rotor is described as folows,

$$
\begin{aligned}
\overrightarrow{J_{\mathrm{sc}}} & =f(T, \vec{B}) \frac{\vec{E}}{|\vec{E}|}, \quad \text { if }|\vec{E}|>E_{\text {zero }} \\
\frac{\partial \overrightarrow{J_{\mathrm{sc}}}}{\partial t} & =0, \quad \text { if }|\vec{E}| \leq E_{\text {zero }} .
\end{aligned}
$$

Here, $T$ and $\vec{B}$ denote temperature and magnetic flux density vector, respectively. $E_{\text {zero }}$ means the criterion for the convergence of the calculation. Function $f$ is obtained by the power law approximation of $E-J$ curve as follows,

$$
f(T, \vec{B},|\vec{E}|)=J_{c}(T, \vec{B})\left[\frac{|\vec{E}|}{E_{0}}\right]^{1 / n(T, \vec{B})} .
$$

Where, $n$ denotes the power law exponent. In this study, however, we assume $J_{c}(T, \vec{B})=J_{c}(T)$ and $n(T, \vec{B})=n(T)$, for simplicity.

By using the vector potential $\vec{A}$, the governing equation is described as follows,

$$
\operatorname{rot}\left(\frac{1}{\mu_{0}} \operatorname{rot} \vec{A}-\vec{M}\right)=\vec{J}_{0}+\sigma \vec{E}+f \frac{\vec{E}}{|\vec{E}|}
$$
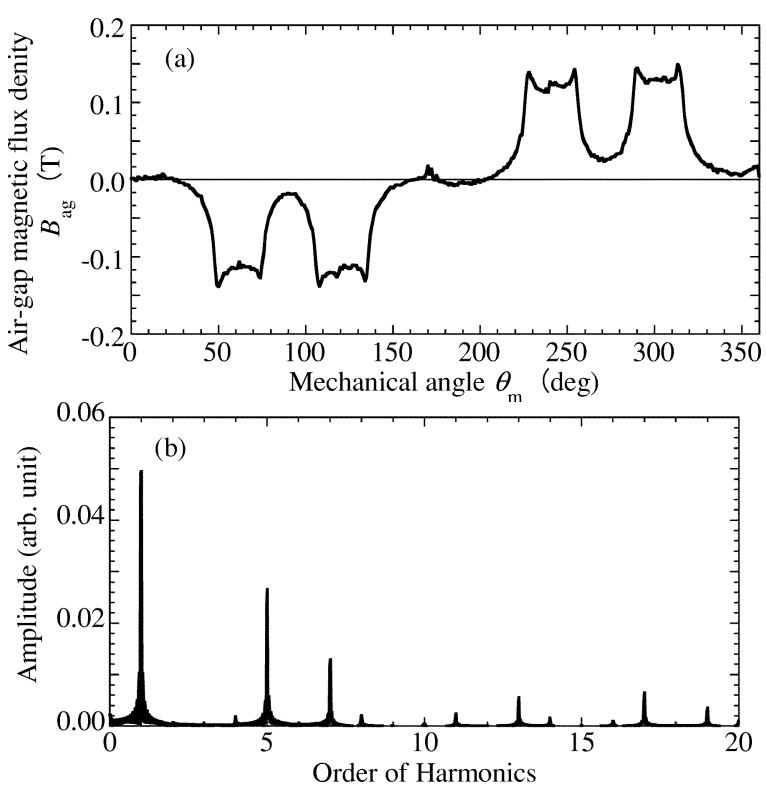

Fig. 4. Experimental results of (a) Magnetic flux density distribution in the air-gap, and (b) corresponding fast fourier transform resuls at room temperature and $2 \mathrm{~A}$ aramature current. Electrical angle of phase-a is $0^{\circ}$.

where, $\mu_{0}$ and $\sigma$ denote, respectively, the permeability in vacuum and the conductivity in normal conductor. $\vec{M}$ is magnetization, and $\vec{J}_{0}$ the forced current density. Then, by taking account of the gauge condition $\vec{E}=-\partial \vec{A} / \partial t$, (4) is described as follows,

$$
\sigma \frac{\partial \vec{A}}{\partial t}+f \frac{1}{\left|\frac{\partial \vec{A}}{\partial t}\right|} \frac{\partial \vec{A}}{\partial t}+\operatorname{rot}\left(\frac{1}{\mu_{0}} \operatorname{rot} \vec{A}-\vec{M}\right)=\vec{J}_{0}
$$

(5) is solved by 3D-FEM. Simulation is carried out for more than one cycle in order to examine the hysteresis property that lie on the major loop.

\section{RESULTS AND DISCUSSION}

\section{A. Distribution of Magnetic Flux Density in Air-Gap at Room Temperature}

Experimental result of the magnetic flux density distribution in the air-gap is shown in Fig. 4(a). Armature current of each phase is set to be $2 \mathrm{~A}$, and the electrical angle of phase-a is $0^{\circ}$. Measurements are performed at room temperature, then the $\mathrm{Bi}-2223$ rotor is in the normal conducting state. As can be clearly seen, the spatial distribution of the flux density is largely distorted due to the concentrated and salient armature windings. Fig. 4(b) shows the corresponding fast fourier transform (FFT) result. Compared to fundamental components, large components of 5th and 7th order harmonics are included. It has already been shown that these harmonic components are one of the main reasons for the slip operation of the fabricated motor [14].

The spatial distribution of the air-gap flux density is reproduced by 3D-FEM analysis in order to see the validity of the model. Fig. 5 shows the simulation results with the same conditions of the experiment. By comparing with Fig. 4, one can see that the 3D-FEM results agree very well with the experiments. 

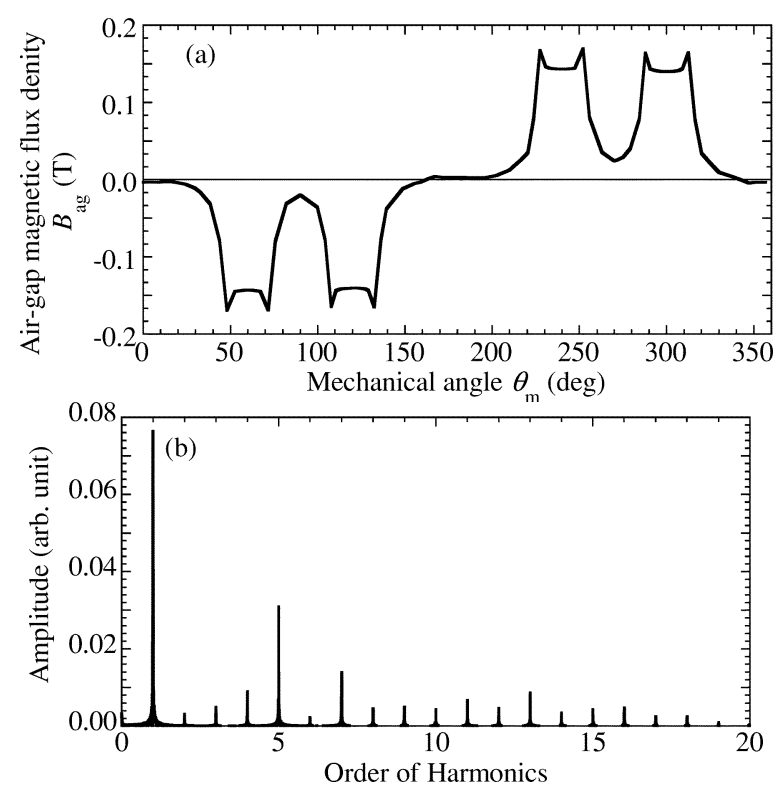

Fig. 5. 3D-FEM analysis results of (a) Magnetic flux density distribution in the air-gap, and (b) corresponding fast fourier transform resuls at room temperature and $2 \mathrm{~A}$ aramature current. Electrical angle of phase-a is $0^{\circ}$.

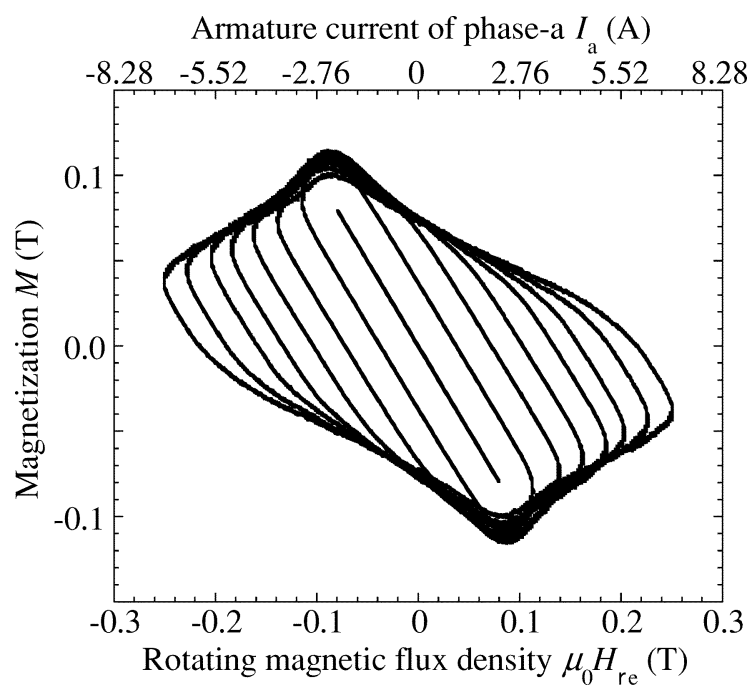

Fig. 6. Experimental results of magnetization, $M$, vs. rotating magnetic flux density, $\mu_{0} H_{\text {re }}$, curves in the air-gap at $77.3 \mathrm{~K}$. The frequency of the rotating field is $60 \mathrm{~Hz}$. The corresponding armature current of phase-a is also shown on the $x$-axis.

\section{B. Distribution of Magnetic Flux Density in Air-Gap at $77.3 \mathrm{~K}$}

The same measurements are also performed with the system that is immersed in liquid nitrogen $(77.3 \mathrm{~K})$. Bi-2223 bulk rotor is now in the superconducting state. Fig. 6 shows the typical experimental results of magnetization, $M$, vs. rotating magnetic flux density, $\mu_{0} H_{\text {re }}$, curves in the air-gap at $60 \mathrm{~Hz}$ frequency [15]. It can be easily deduced that these hysteresis characteristics influence the aforementioned harmonics of the spatial magnetic flux distribution. Typical contour plots of magnetic flux density distribution obtained by 3D-FEM is shown in Fig. 7. The critical current density, $J_{c}$ and $n$ value are, respectively, $1 \times 10^{7} \mathrm{Am}^{-2}$ and 10.0 at $77.3 \mathrm{~K}$. With these results, the distribution of magnetic flux density on the surface of the bulk is

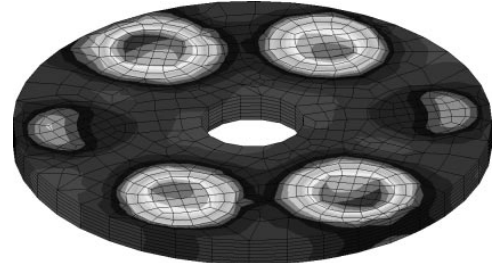

Fig. 7. 3D-FEM analysis result of magnetic fluc density contour plot of $\mathrm{Bi}-2223$ bulk rotor in a rotating magnetic field at $77.3 \mathrm{~K}$.
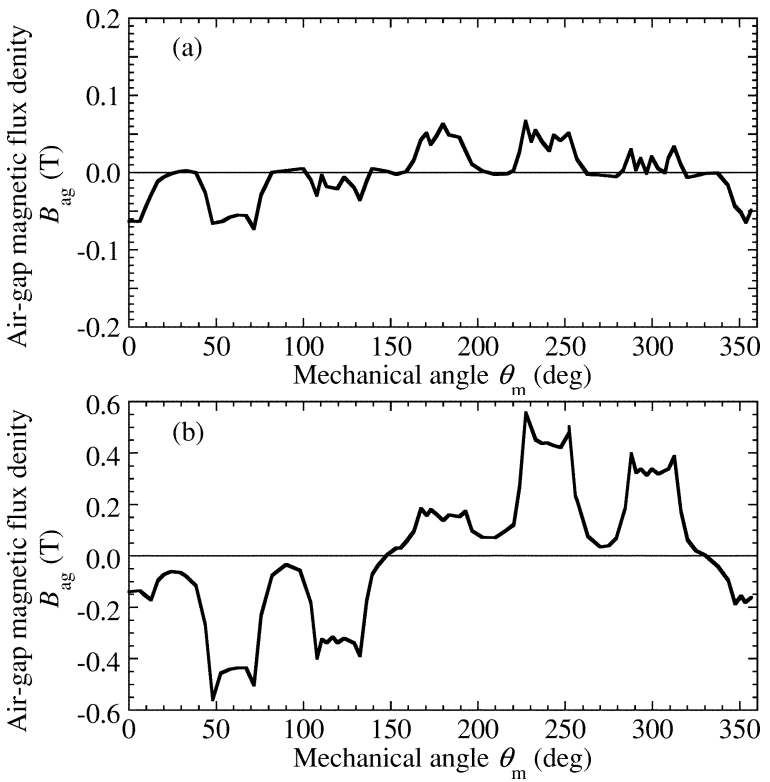

Fig. 8. 3D-FEM analysis results of magnetic flux density distribution in the air-gap at $77.3 \mathrm{~K}$ for (a) $2 \mathrm{~A}$ and (b) $6 \mathrm{~A}$, respectively. Electrical angle of phase-a is $0^{\circ}$.

calculated with the same condition of the experiment. Fig. 8 shows the calculated results for the armature current of (a) 2.0 A and (b) 6.0 A. As compared with the results obtained for the rotor in the normal conducting state (Fig. 5(a)), the spatial distribution is more severely distorted due to the shielding properties of the superconducting rotor (Fig. 8(a)). When the armature current increases, however, more flux penetrates, and then the amplitude of the rotating field is recovered (Fig. 8(b)). These results are semi-quantitatively consistent with the experimental data [15]. Normalized harmonic components with fundamental one are listed in Table I. The corresponding experimental results are also shown in Table II [15]. From these results, one can say that the shielding property of the HTS bulk increases the 5th and 7th components of harmonics especially at the lower rotating magnetic field at which the flux penetrates only partially into the bulk rotor. It is well known that these harmonic components bring about a detrimental effect on the performance of the motor [13]-[16]. Therefore, the flux has to be fully penetrated in order to realize the full performance of the motor.

The quantitative description of the magnetic characteristics is not available because the magnetic field dependency of the critical current density is not considered in this study. As can be seen from the shape of the hysteresis loops shown in Fig. 6, this dependency has to be taken into account [17], and will be our next study for more precise discussion. 
TABLE I

3D-FEM RESULTS OF THE HARMONIC COMPONENTS

\begin{tabular}{lccc}
\hline \hline Harmonics & Amplitude ratio & & \\
& 2 A,room temperature & $2 \mathrm{~A}, 77.3 \mathrm{~K}$ & $6 \mathrm{~A}, 77.3 \mathrm{~K}$ \\
\hline 1st & 1 & 1 & 1 \\
5th & 0.405 & 0.596 & 0.603 \\
7th & 0.182 & 0.389 & 0.194 \\
\hline \hline
\end{tabular}

TABLE II

EXPERIMENTAL RESULTS OF THE HARMONIC COMPONENTS

\begin{tabular}{lccc}
\hline \hline Harmonics & $\begin{array}{c}\text { Amplitude ratio } \\
\text { 2 A,room temperature }\end{array}$ & $2 \mathrm{~A}, 77.3 \mathrm{~K}$ & $6 \mathrm{~A}, 77.3 \mathrm{~K}$ \\
\hline 1st & 1 & 1 & 1 \\
5th & 0.534 & 0.666 & 0.581 \\
7th & 0.268 & 0.364 & 0.272 \\
\hline \hline
\end{tabular}

\section{CONCLUSION}

Spatial distribution of the air-gap magnetic flux density was measured in a rotating magnetic field at $60 \mathrm{~Hz}$ frequency. 3D-FEM analysis was also carried out in order to understand the effect of pinning property of Bi-2223 bulk rotor upon the harmonic components of the air-gap flux distribution. It was shown that the hysteresis property of the bulk influences the harmonic components when the magnetic flux is partly penetrated. How- ever, the harmonics were reduced in the case where the flux is fully penetrated.

\section{REFERENCES}

[1] L. K. Kovalev, K. V. Ilushin, V. T. Penkin, K. L. Kovalev, S. M. -A. Koneev, V. A. Modestov, S. A. Larionoff, W. Gawalek, and B. Oswald, "HTS electrical machines with YBCO bulk and Ag-BSCCO plate-shape HTS elements: Recent results and future development," Physica $C$, vol. 354, pp. 34-39, 2001.

[2] L. K. Kovalev, K. V. Ilushin, V. T. Penkin, K. L. Kovalev, S. M. -A. Koneev, K. A. Modestov, S. A. Larionoff, W. Gawalek, and B. Oswald, "Electrical machines with bulk HTS elements. The achieved results and future development," Physica C, vol. 357-360, pp. 860-865, 2001.

[3] M. D. McCulloch and D. Dew-Hughes, "Brushless ac machines with high temperature superconducting rotors," Materials Science and Engineering, vol. B53, pp. 211-215, 1998.
[4] M. D. McCulloch, G. J. Barnes, and D. Dew-Hughes, "Application of high temperature superconductors to electrical machines," in Proceedings of International Conference on Electrical Machines 2000, Espoo, Finland, 2000, pp. 812-816.

[5] L. K. Kovalev, K. V. Ilushin, V. T. Penkin, K. L. Kovalev, S. M. -A. Koneev, V. N. Poltavets, A. E. Larionoff, K. A. Modestov, S. A. Larionoff, W. Gawalek, T. Habisreuther, B. Oswald, K. -J. Best, and T. Strasser, "Hysteresis and reluctance electric machines with bulk HTS elements. Recent results and future development," Superconductor Science and Technology, vol. 13, pp. 498-502, 2000.

[6] D. Dew-Hughes, M. D. McCulloch, K. Jim, J. Aldwinckle, G. J. Barnes, G. Jones, J. R. Gaines Jr, and S. Sengupta, "Liquid cryogen pumps integrated with superconducting motors," Advances in Cryogenic Engineering, 2000.

[7] T. Habisreuther, T. Strasser, W. Gawalek, P. Görnert, K. V. Ilushin, and L. K. Kovalev, "Magnetic processes in hysteresis motors equipped with melt-textured YBCO," IEEE Transactions on Applied Superconductivity, vol. 7, pp. 900-903, 1997.

[8] L. K. Kovalev, K. V. Ilushin, V. T. Penkin, K. L. Kovalev, V. S. Semenikhin, V. N. Poltavets, A. E. Larionoff, W. Gawalek, T. Habisreuther, T. Strasser, A. K. Shikov, E. G. Kazakov, and V. V. Alexandrov, "Hysteresis electrical motors with bulk melt-textured YBCO," Materials Science and Engineering, vol. B53, pp. 216-219, 1998.

[9] H. Ohsaki and Y. Tsuboi, "Study on electric motors with bulk superconductors in the rotor," Journal of Materials Processing Technology, vol. 108, pp. 148-151, 2001.

[10] Y. Tsuboi and H. Ohsaki, "Electromagnetic phenomena in bulk superconductors applied in a rotating machine during transient state," Physica $C$, vol. 357-360, pp. 889-891, 2001.

[11] I. Muta, H. J. Jung, T. Hirata, T. Nakamura, T. Hoshino, and T. Konishi, "Fundamental experiments of axial-type BSCCO-bulk superconducting motor model," IEEE Transactons on Applied Superconductivity, vol. 11, pp. 1964-1967, 2001.

[12] I. Muta, T. Nakamura, T. Hirata, T. Hoshino, and T. Konishi, "Preliminary study on axial-type BSCCO superconducting motor," Physica C, vol. 354, pp. 100-104, 2001.

[13] I. Muta, H. J. Jung, T. Nakamura, and T. Hoshino, "Performance of axial-type motor with Bi-2223 HTS bulk rotor," Physica C, vol. 372-375, pp. 1535-1538, 2002.

[14] - "Performances of axial-type superconducting BSCCO-2223 motor," in Proceedings of International Conference on Electrical Engineering 2002, 2002, pp. 190-195.

[15] T. Nakamura, I. Muta, H. J. Jung, and T. Hoshino, "Electromagnetic characteristics of axial-type HTS motor utilizing a Bi-2223 bulk rotor" (in Japanese), Cryogenic Engineering, vol. 37, no. 11, pp. 726-733, 2002.

[16] H. J. Jung, T. Nakamura, I. Muta, and T. Hoshino, "Characteristics of axial-type HTS motor under different magnetic and temperature conditions," in Preprint of Applied Superconductivity Conference 2002, 4LF03.

[17] — "Superconductor disks and cylinders in an axial magnetic field. I. flux penetration and magnetization curves," Physical Review B, vol. 58, no. 10 , pp. 6506-6522, 1998. 